\title{
Reconstruction of Pilonidal Sinus by Rhomboid Flap : Our Experience
}

\author{
Md. Saiful Hoque ${ }^{1 *}$ \\ Tahmina Akther Chowdhury ${ }^{2}$ \\ Saifuddin Ahmed Siddique ${ }^{3}$ \\ Md. Saiful Islam
}

'Department of Surgery

Chittagong Medical College

Chittagong, Bangladesh.

${ }^{2}$ Department of Paediatric Surgery

Chittagong Medical College

Chittagong, Bangladesh.

${ }^{3}$ Department of Urology

Chattagram Maa-O-Shishu Hospital Medical College

Chittagong, Bangladesh.
${ }^{*}$ Correspondence to:

Dr. Md. Saiful Hoque

Associate Professor

Department of Surgery

Chittagong Medical College

Chittagong, Bangladesh.

Mobile: +8801556322867

E-mail: saifulssurgery@yahoo.com

www.banglajol.info/index.php/CMOSHMCJ

\begin{abstract}
Background: Pilonidal sinus is a common disease of young adult usually caused by insertion of fallen hair into skin (Gluteal cleft). Wide excision surgery is a common practice but due to high recurrence and long duration of healing there are more simple alternatives. Our experience with transposition of Rhomboid flap in the treatment of pilonidal sinus are described. The conventional way of treatment of pilonidal sinus is block excision and lay open of the sinus result in 5-14 days hospital stay healing time of 6-10 weeks. In our study all patients hospital stay was 2-3 days, healing time was less than two weeks with minimum complication. The aim of the study is to do rhomboid flap for the treatment of pilonidal sinus, so to avoid complications and recurrence, to shorten hospital stay and to give better outcome. Methods: Total 6 patients of 18-40 years of age after doing all investigations and clinical examinations, excision of pilonidal sinus was done and repaired by rhomboid transposition flap in the same setting. All the patients were followed up post operatively for 3 months to see any discharging sinus. Results: All 6 patients were discharged on $3^{\text {rd }}$ post operative day and stitches were removed on $8^{\text {th }}$ post operative day and approximate time to resume their work is 14 days Conclusion: Pilonidal sinus surgery is a challenging operation for the surgeon because of recurrence and complication. Rhomboid flap technique has become familial because of its advantage of early healing, less hospital stay and very low recurrence rate.
\end{abstract}

Key words: Pilonidal sinus; Rhomboid flap; Primary healing.

\section{INTRODUCTION}

Pilonidal sinus is a chronicsubcutaneous abscess in the natal cleft, which spontaneously drains through the opening. A.W Anderson's letter to the editor that appeared in an issue of the Boston Medical Surgical Journal of 1847, entitled 'Hair extracted from an ulcer' is believed to be the first documented case of pilonidal sinus. In 1880, Hodges coined the term 'pilonidal sinus' to describe the chronic sinus containing hair and found between the buttock ${ }^{1}$. This pilonidal sinus disease was also branded as "jeep disease" because of the high incidence among jeep drivers ${ }^{1,2}$. The onset of pilonidal sinus disease is rare both before puberty and after the age of 40.Males are more affected than females. The average age of presentation is 21 years for male and 19 years for female. The risk factors and associations include sedentary occupation, positive family history, obesity, local irritation and trauma ${ }^{3}$.

The origin of pilonidal disease is not fully understood. There are two theories associated with its pathogenesis: the acquired and the congenital theories. However, the majority of opinion favours the acquired theory, which postulates that the sacrococcygeal pilonidal infection originates in a natal cleft hair follicle that has become distended with keratin leading to folliculitis the formation of abscess that extends down into the subcutaneous tissue. Then hair can enter through the tiny pit and lodge in the abscess cavity from the suction created by the movement of the gluteal region ${ }^{1}$. Hormone, hair, friction and infection all play a role in the pathogenesis of the disease ${ }^{3}$. 
Pilonidal disease is a common medical condition that accounts for almost $15 \%$ of anal suppurations ${ }^{2}$. Many methods of treatment have been advocated to manage this disease including incision and drainage of acute pilonidal abscess, excision and healing by open secondary intention, excision and partial closure, excision and primary closure and z-plasty, Karydakis flap procedure, Rhomboid transposition of flap, Bascom's flap, Gluteus maximusmyocutaneous flap.In spite of multiple surgical technique recurrence is common ${ }^{1,4}$.

\section{MATERIALS AND METHODS}

In this study 6 patients were included with chronic pilonidal sinus. The age was between 18 and 40 years. Detailed history was taken and thorough clinical examination was done to exclude other conditions that resemble pilonidal sinus. All routine investigations done for all patients. All the patients were treated surgically by excision of sinus and primary repair by Rhomboid transposition flap. All the patients were advised to lay in right or left lateral position and to use high commode. All the patients were discharged from hospital at $3^{\text {rd }}$ postoperative day after check dressing and stitch off done at $8^{\text {th }}$ postoperative day. They were followed up after three months as outpatient clinic. Long term follow up was by personal review at a mean time of 8 months.

All the patients were operated under spinal anaesthesia placing them in jack knife position. The buttocks are meticulously shaved. A vertical line ac was drawn at the midline including the pilonidal opening measuring about $10 \mathrm{~cm}$. A rhomboid is outlined to encompass the pilonidal sinuses in the midline. The lines were $a b=b c=c d=a d$ all of which were $60 \%$ of $a c$. The line de is drawn to bisect the angle made by bd and cd. The lines de and ef are the same length as ad. The d-e-f angle is the same as the b-a-d angle. The rhomboid excision is made down to the sacral periosteum in the midline and to the gluteal muscle laterally. The d-e-f flap is made including skin, subcutaneous tissueand muscle fascia. The flap is then transposed to cover the rhomboid wound where e is placed at a and $\mathrm{f}$ is placed at $\mathrm{d}$ point and the wound is closed with suture.

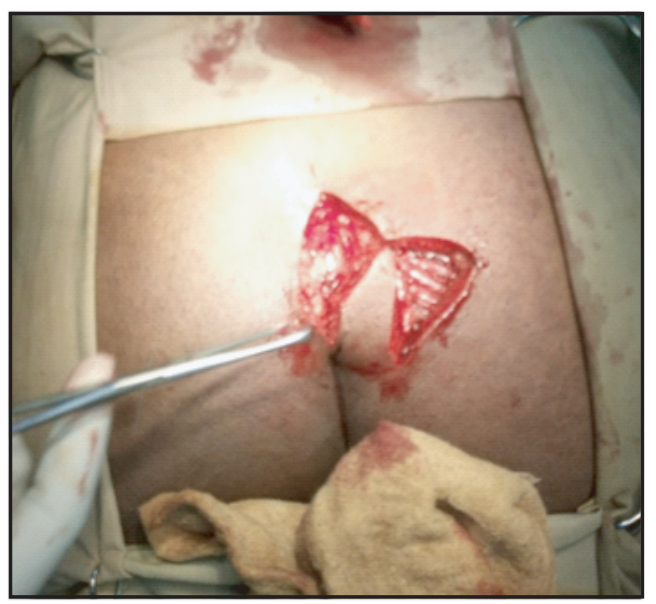

Figure 1: After dissection of rhomboid flap.

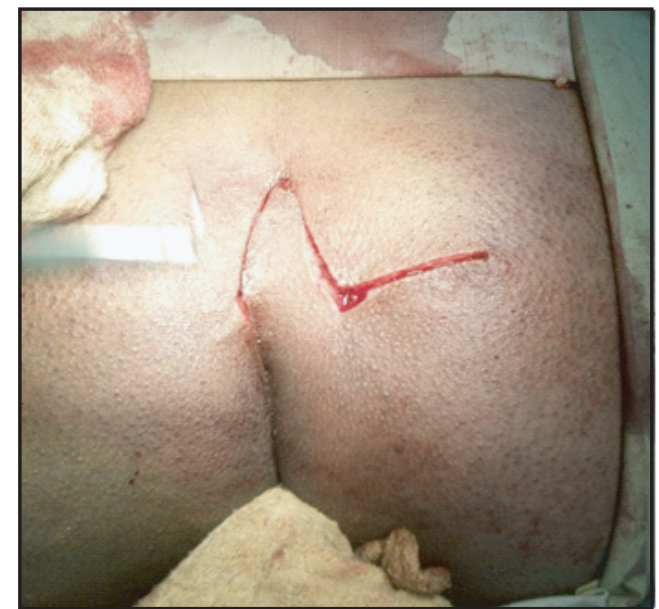

Figure 2 : After transposition of flap to cover the rhomboid wound.

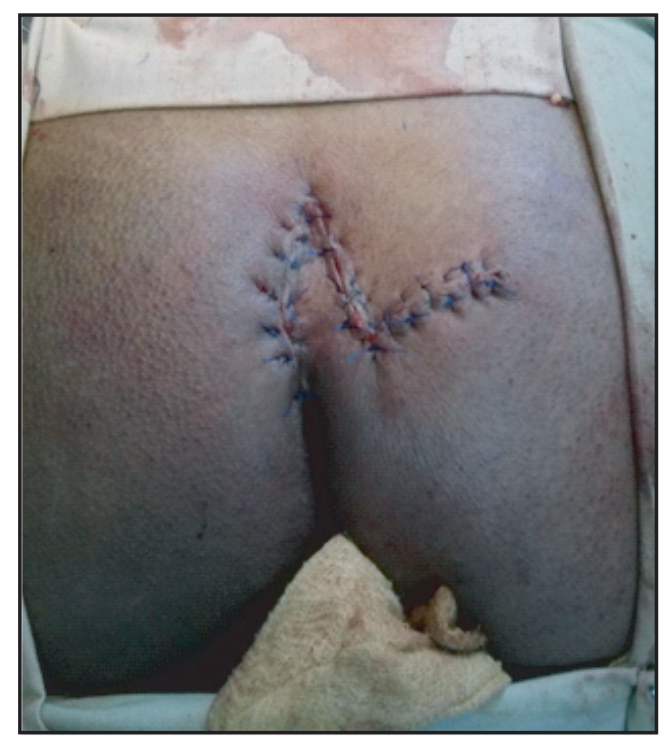

Figure 3 : After closure of skin.

\section{RESULTS}

All six operations healed by primary intention with no episodes of wound sepsis.All the patients were kept hospitalized for 2 days after operation. They were discharged at $3^{\text {rd }}$ postoperative day and stitch off done at $8^{\text {th }}$ postoperatve day. They were allowed to return to their occupations 14 days after operation. Long term follow up was by personal review at a mean time of 8 months (Range 6-10 months) after surgery. During this time no recurrence cases had been observed.

\section{DISCUSSION}

Pilonidal sinus can be a chronic and recurring condition which is sometimes difficult to cure.There are many operative techniques for the management of it. In this study we used rhomboid flap method for chronic pilonidal sinus. Oueidat D et al, in their study showed that in block excision and lay open of 
the sinuses result in hospital stay of 5 - 14 days, healing times of 6 - 10 weeks and various other methods of primary closure reduced the healing time to less than 2 weeks, but hospitalization remained almost the same between 7 to 10 days $^{2}$. Gwynn B R, in his study of 20 patients, operated by Rhomboid flap method, had observed no wound sepsis but one recurrence ${ }^{5}$. In our study of six patients, hospital stay is only 2 days, average healing time less than two weeks and we have found no postoperative complications.

\section{CONCLUSION}

Pilonidal sinus still now a challenging operation for the surgeons because of its high recurrence rate. Selection of operative technique depends on the surgeon's familiarity with the procedure and the perceived results in terms of healing and recurrence rates. Now a day's rhomboid flap technique has become familiar because of its advantage of early healing, less hospital stay and lower recurrence rate.

DISCLOSURE

All the authors declared no competing interest.

\section{REFERENCES}

1. Gordon P.H, Nivatvongs S. Pilonidal disease. In: Cataldo P.A, Macdonald J, Schouten W.R, Smith L.E,editors. Principles and practice of surgery for the colon, rectum and anus. Third edition. New York: Informa healthcare. 2007; 235-246.

2. D. Oueidat et al. Open Journal of Gastroenterology. 2014;4:1-5.

3. Z.Matar. Pilonidal sinus disease: A 5-year study. The internet journal of surgery.2006;13(2).

4. Romero C.J, Alcalde H, Martin F, Pulido A, Rico P. Treatment of pilonidal sinus by excision rhomboid flap. Int J Colorectal Dis. 1990;5:200-202.

5. Gwynn B.R. Use of the Rhomboid flap in pilonidal sinus. Annals of the Royal college of surgeons of England.1986; 68. 